\title{
Design of Choke Inductor in Class-E ZVS Power Amplifier
}

\author{
Agasthya Ayachit, Dalvir K. Saini, and \\ Marian K. Kazimierczuk \\ Department of Electrical Engineering \\ Wright State University \\ 3640 Colonel Glenn Hwy., Dayton, OH, 45435, USA \\ \{ayachit.2, saini.11, marian.kazimierczuk\}@wright.edu
}

\author{
Alberto Reatti \\ Department of Information Engineering (DINFO) \\ University of Florence \\ Via Santa Marta 3 I-50139 Florence \\ alberto.reatti@unifi.it
}

\begin{abstract}
This paper presents the following for a Class-E zero-voltage switching (ZVS) power amplifier: (a) design of the choke inductor and (b) theoretical estimation of power losses in the core and a solid round winding. The expressions required to design the core using the Area-Product $\left(A_{p}\right)$ method are provided. The equations for the dc resistance, ac resistance at high frequencies, and dc and ac power losses are provided for the solid round winding. A Class-E ZVS power amplifier with practical specifications is considered. A core with air gap is selected since the choke inductor carries a dc current in addition to the ac component. The gapped core power loss density and power loss are estimated using Steinmetz empirical equation. Simulation results showing the transient analysis and Fourier analysis are given. It is shown that, for the given design, the winding power loss due to the fundamental component is dominant and that due to higher order harmonics can be neglected. In addition, it is also proven that the power loss caused by the dc current component is higher than that by the ac current component, which can be neglected.
\end{abstract}

\section{INTRODUCTION}

Magnetic components are integral parts in many electronic circuits [1] - [6]. An ideal choke must reject any ac component and conduct only the dc component. However, in practical applications, the choke inductor conducts a fraction of ac current in addition to the dc component and the ac component can reach high-frequenies and its waveform is usually not sinusoidal. . Thus, the design procedure must consider both these components and predict the nature of losses in the core and the winding. This paper presents a comprehensive procedure to design the choke inductors used in Class-E ZVS power amplifiers. Class-E ZVS power amplifiers belong to a class of highly efficient electronic circuits. Therefore, the component design must be optimized to yield the maximum efficiency.

The magnetic core for the choke inductor is designed using the Area-Product $A_{p}$ method. This is a widely adopted method to magnetic components in pulse-width modulated converters as well as ac resonant inductors [2]. Since a majority of the current is dc, air gap is essential to avoid core saturation. In addition, the winding power loss depends on the winding geometry. In this paper, a solid round winding is used and its dc and ac winding power loss are estimated to determine the dominant power loss. Typically, in switching circuits, the current through the choke inductors are non-sinusoidal. The losses in the windings as well as in the magnetic core as caused by the dc, fundamental, and higher order harmonics. Therefore, Fourier analysis is performed and the magnitude of the harmonic components are evaluated.

The objectives of this paper are as follows:

1) To develop a theoretical framework design the choke inductance for a Class-E zero-voltage switching power amplifier.

2) To present the design equations necessary to calculate the dc and ac resistances and dc and ac power losses for the choke inductor made up of solid round winding.

3) To provide the necessary expressions required to design the magnetic gapped core using Area-Product method.

4) To evaluate the fundamental and higher order harmonic components of the choke inductor current and determine their power losses.

5) To verify the presented analysis through circuit simulations.

\section{Design EQUATIONS}

\section{A. Design of Class-E ZVS Power Amplifier}

Fig. 1 shows the circuit of the Class-E zero-voltage switching (ZVS) power amplifier. The amplifier is supplied by a dc input voltage source $V_{I}, L_{f}$ is the choke inductance, $S$ is the power MOSFET, $C_{1}$ is the voltage-shaping shunt capacitance, which includes the output capacitance of the MOSFET, $L$ and $C$ form the resonant circuit inductor and capacitor, while $R$ is the load resistance.

The expression for the load resistance is

$$
R=\frac{8}{\pi^{2}+4} \frac{V_{I}^{2}}{P_{O}},
$$

where $P_{O}$ is the output power. The choke inductor can be designed using

$$
L_{f}=2\left(\frac{\pi^{2}}{4}+1\right) \frac{R}{f_{s}}
$$




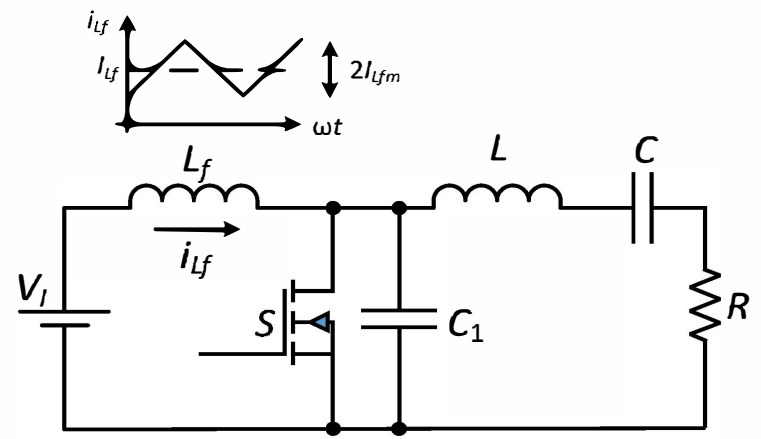

Fig. 1. Circuit of the Class-E power amplifier.

where $f_{s}$ is the switching frequency of the MOSFET. The dc current flowing through the choke is equal to the input current and is expressed as

$$
I_{L f}=I_{I}=\frac{P_{I}}{V_{I}}=\frac{P_{O}}{\eta V_{I}},
$$

where $\eta$ is the overall efficiency of the Class-E amplifier. The choke inductor carries an ac component in addition to the dc current. The amplitude of the ac current through the choke is given by

$$
I_{L f m}=\frac{V_{I}}{4 f_{s} L_{f}} .
$$

Therefore, the peak value of the choke inductor current is

$$
I_{L f(\max )}=I_{L f}+I_{L f m}=\frac{P_{O}}{\eta V_{I}}+\frac{V_{I}}{4 f_{s} L_{f}} .
$$

The choke inductor must be designed to withstand the peak current $I_{L f(\max )}$. The winding carrying the choke inductor current must satisfy the current density requirement as well as exhibit low ac power losses.

The current through the choke inductor can be approximated by a symmetrical triangular wave of magnitude $I_{L f m}$ as shown in Fig. 2. The Fourier series expansion of the triangular wave is

$$
i_{L f}=\frac{8 I_{m n}}{\pi^{2}} \sum_{n=1,3,5, \ldots}^{\infty} \frac{(-1)^{(n-1) / 2}}{n^{2}} \sin \left(n \omega_{s} t\right) .
$$

The fundamental component of the triangular waveform is obtained by substituting $n=1$ in (6) to get

$$
i_{L f 1} \approx I_{m 1} \sin \omega_{s} t
$$

where $\omega_{s}$ is the angular switching frequency and the amplitude of the fundamental of the ac component through the choke inductor is

$$
I_{m 1}=\frac{8 I_{L f m}}{\pi^{2}} .
$$

In (8), $I_{L f m}$ is the peak value of the triangular waveform of the choke inductor current. By substituting $n=3$ in (6), the third harmonic component is

$$
i_{L f 3} \approx I_{m 3} \sin \left(3 \omega_{s} t\right)
$$

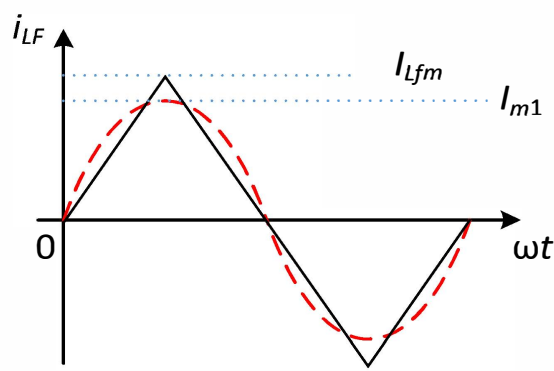

Fig. 2. Waveforms of the ac component of the choke inductor current and its fundamental harmonic component.

and the amplitude of the third harmonic component is

$$
I_{m 3}=\frac{8 I_{L f m}}{9 \pi^{2}}=\frac{I_{m 1}}{9} .
$$

The even harmonics $(n=2,4,6, \ldots)$ are zero since the triangular waveform is assumed to be symmetric about its dc value. By expanding the series, it can be noted that the amplitudes of the harmonics for $n \geq 3$, i.e., $I_{m 3}, I_{m 5}, \ldots$ are much lower than the amplitude of the fundamental $I_{m 1}$. Therefore, the effect of the higher order harmonics of the choke inductor current on the winding and core power can be neglected.

\section{B. Design of Core Using Area-Product Method}

The area-product method relates the geometrical core parameters described by the core manufacturers with the inductor magnetic and electric parameters [3]. The maximum energy stored in the choke inductor due to both dc and ac currents is

$$
W_{L f}=\frac{1}{2} L_{f} I_{L f(\max )}^{2} \text {. }
$$

The window utilization factor $K_{u}$ and the saturation flux density $B_{\text {sat }}$ are assumed a priori. The current density of the conductor is selected based on the peak current requirement and is discussed in the following section. The core areaproduct is given by

$$
A_{p}=W_{a} A_{c}=\frac{4 W_{L f}}{K_{u} J_{m} B_{s a t}},
$$

where $W_{a}$ is the core window area and $A_{c}$ is the core cross-sectional area, both of which are mentioned in the core manufacturer datasheet. Further, the window area is estimated from (12) as

$$
W_{a}=\frac{A_{p}}{A_{c}} .
$$

From the selected core geometry, the number of turns $N$ is determined using a specified window area $W_{a}$, window utilization factor $K_{u}$, and solid round wire current density as $J_{m}$ as

$$
N=\frac{W_{a} K_{u} J_{m}}{2 I_{L f(\max )}}
$$

However, for the gapped core, to achieve the desired inductance and strength of magnetization, the number of turns is 
higher than that calculated in (14). Thus, for a core with air gap with a predetermined inductance $L_{f}$, the number of turns is [3]

$$
N=\sqrt{\frac{L_{f}}{\mu_{0} A_{c}}\left(l_{g}+\frac{l_{c}}{\mu_{r c}}\right)},
$$

where $A_{c}$ is the cross-sectional area of the core, $\mu_{0}$ is the permeability of free-space, $\mu_{r c}$ is the relative permeability of the core material, $N$ is the number of turns, $l_{c}$ is the mean magnetic path length, $l_{g}$ is the assumed length of the air gap. Thus, the actual number of turns is

$$
N=\max \left\{\frac{W_{a} K_{u}}{2 A_{w(s o l)}}, \sqrt{\frac{L_{f}}{\mu_{0} A_{c}}\left(l_{g}+\frac{l_{c}}{\mu_{r c}}\right)}\right\} .
$$

Alternatively, low iron powder cores with a low relative permeability $\mu_{r}$ can be utilized. However, in both of the cases the core permeability is reduced, while the number of turns required to achieve a certain inductance is increased. This affects the winding design. The dc current through the choke as well as the ac current are functions of the load resistance of the amplifier. In several applications, for example, Class-E ZVS inverter in wireless power charging systems, the load resistance is a function of the distance between the transmission coil and the receiver coil. In such operating conditions, the dc and ac current may exceed a critical value causing the core to saturate. This requires an air gap in the core. For the gapped core, the peak value of the magnetic flux density in the core of the choke inductor is given as

$$
B_{p k}=\frac{\mu_{r c} \mu_{0} N I_{L f(\max )}}{l_{c}\left(1+\frac{\mu_{r c} l_{g}}{l_{c}}\right)} .
$$

However, the amplitude of magnetic flux density due to the fundamental of the ac component of the choke inductor current is

$$
B_{c m}=\frac{\mu_{r c} \mu_{0} N I_{m 1}}{l_{c}\left(1+\frac{\mu_{r c} l_{g}}{l_{c}}\right)}=\frac{8 \mu_{r c} \mu_{0} N I_{L f m}}{\pi^{2} l_{c}\left(1+\frac{\mu_{r c} l_{g}}{l_{c}}\right)} .
$$

Thus, for the gapped core, the Steinmetz empirical equation for the power loss density at frequency $f_{s}$ is [4], [5]

$$
P_{v g}=k f^{a} B_{c m}^{b}=k f_{s}^{a}\left[\frac{8 \mu_{r c} \mu_{0} N I_{L f m}}{\pi^{2} l_{c}\left(1+\frac{\mu_{r c} l_{g}}{l_{c}}\right)}\right]^{b},
$$

where $k, a$, and $b$ are the Steinmetz empirical constants [4]. Consequently, the total power loss in the gapped core is

$$
\left.P_{C g}=P_{v g} V_{C}=k f_{s}^{a}\left[\frac{8 \mu_{r c} \mu_{0} N I_{L f m}}{\pi^{2} l_{c}\left(1+\frac{\mu_{r c} l_{g}}{l_{c}}\right)}\right]\right]^{b} A_{C} l_{c} .
$$

\section{Winding Design}

This section provides a methodology to design the inductor with a solid round winding. The solid round winding is designed to withstand the maximum current stress at a uniform

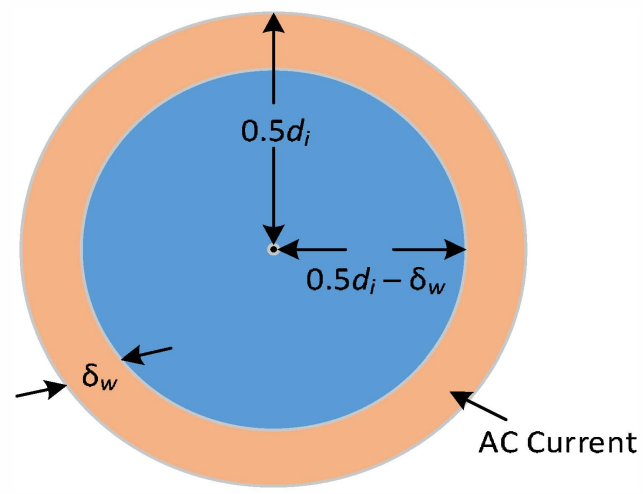

Fig. 3. Solid round conductor showing a reduced effective area available for current conduction at high frequencies due to skin-effect.

dc current density. Assuming a copper conductor, the skin depth as a function of frequency is [3],

$$
\delta_{w}=\sqrt{\frac{\rho}{\pi f_{o} \mu_{r} \mu_{0}}}=\frac{66.2}{\sqrt{f_{o}}},
$$

where $\rho=1.724 \times 10^{-8} \Omega \cdot \mathrm{m}$ is the resistivity of the conductor, $f_{o}$ is the operating frequency, $\mu_{r}$ is the relative permeability of the conductor $\left(\mu_{r}=1\right.$ for metals), and $\mu_{0}$ is the absolute permeability of free space. Let $J_{m}$ be the current density of the winding. For the selected choke inductor, current density is given by

$$
J_{m} \approx \frac{I_{L f(\max )}}{A_{w(s o l)}} \approx \frac{I_{L f}}{A_{w(s o l)}},
$$

where $J_{m}$ is in $\mathrm{A} / \mathrm{mm}^{2}$ and $A_{w(s o l)}$ is the cross-sectional area of the winding. Thus, the inner diameter of the solid round winding must be

$$
d_{i(s o l)}=\sqrt{\frac{4 A_{w(s o l)}}{\pi}} \geq 2 \delta_{w} .
$$

A corresponding wire for the inductor winding is chosen from the AWG datasheet. Its inner and outer diameters are noted along with the bare wire area and the resistance per unit length. In addition, the average length per turn $l_{T}$ of the winding around the core is also estimated. Thus, the total length of the winding is $l_{w}=N l_{T}$. The dc resistance of the solid round winding is

$$
R_{w d c(s o l)}=\frac{\rho l_{w}}{A_{w(s o l)}}=\frac{4 \rho N l_{T}}{\pi d_{i(s o l)}^{2}} .
$$

The conduction power loss due to the dc component is

$$
P_{R w d c(s o l)}=I_{L f}^{2} R_{w d c(s o l)}=\frac{4 \rho N l_{T} I_{I}^{2}}{\pi d_{i(s o l)}^{2}},
$$

At high operating frequencies, the available area for current conduction is smaller than that at dc or low frequencies. This phenomena occurs due to the skin effect phenomena at high frequencies, thereby increasing the overall resistance of the 
winding [3]. This effect is illustrated in Fig. 3. The effective area is determined as

$$
A_{e}=\frac{\pi d_{i}^{2}}{4}-\frac{\pi\left(d_{i}-\delta_{w}\right)^{2}}{4}=\pi \delta_{w}\left(d_{i}-\delta_{w}\right) .
$$

Therefore, the solid round winding ac resistance can be approximated by

$$
R_{w a c(s o l)}=\frac{\rho l_{w}}{A_{e}}=\frac{4 \rho N l_{T}}{\delta_{w}\left(d_{i}-\delta_{w}\right)} .
$$

The power loss due to the ac component

$$
P_{w a c(s o l)}=\frac{I_{m 1}^{2}}{2} R_{w a c(s o l)}=\frac{64 I_{L f m}^{2}}{2 \pi^{4}} R_{w a c(s o l)} .
$$

The number of layers is a factor, is related to the area product $A_{p}$ and the window utilization factor $K_{u}$. The selected core allows for a single layer winding to be wound, and, therefore, the proximity effect is neglected in this paper.

\section{Total Power Losses}

For the inductor with solid round winding, the total power loss in the designed inductor is the sum of the core power loss given in (20), the power loss due to dc current given in (25), and the winding loss given in (28), and is expressed as

$$
P_{L f(s o l)}=P_{C g}+P_{w(s o l)}+P_{w a c(s o l)} \text {. }
$$

\section{Design EXAMPLE}

\section{A. Selection of Magnetic Core and Winding}

A choke inductor is designed for the following specifications: supply voltage $V_{I}=10 \mathrm{~V}$, output power $P_{O}=10 \mathrm{~W}$, switching frequency $f_{s}=1 \mathrm{MHz}$. The efficiency of the amplifier is assumed as $\eta=0.9$. Using (1) and (2), the values of the load resistance is $R=5.76 \Omega$ and the choke inductance is $L_{f}=40 \mu \mathrm{H}$. The dc input current using (3) is $I_{I}=I_{L f}=1.11 \mathrm{~A}$. The amplitude of the ac current through the choke inductance using (4) is $I_{L f m}=0.0625 \mathrm{~A}$. Therefore, the peak value of the choke inductor current is $I_{L f(\max )}=I_{L f}+I_{L f m}=1.1725$ A. It must be noted that $I_{L f m} / I_{L f}=5 \%$. Thus, the magnitude of the ac current ripple through the choke inductor is only $5 \%$ of its dc value. The maximum energy stored in the choke inductance using (11) is

$$
W_{L f}=\frac{1}{2} L_{f} I_{L f(\max )}^{2}=\frac{40 \times 10^{-6} \times 1.2^{2}}{2}=0.0288 \mathrm{~mJ} \text {. }
$$

Assume the following core parameters: current density $J_{m}=$ $5 \mathrm{~A} / \mathrm{mm}^{2}$, window utilization factor $K_{u}=0.25$, saturation magnetic field density $B_{\text {sat }}=0.25 \mathrm{~T}$. The core area product using (12) is

$$
A_{p}=\frac{4 W_{L f}}{K_{u} J_{m} B_{\text {sat }}}=\frac{4 \times 0.028 \times 10^{-3}}{0.25 \times 5 \times 10^{6} \times 0.25}=0.0364 \mathrm{~cm}^{4} \text {. }
$$

A Magnetics ${ }^{\circledR}$ ferrite HS gapped pot P-type core 41811 was selected, which has:

- Core area product $A_{p}=0.05 \mathrm{~cm}^{4}$.

- Core cross-sectional area $A_{c}=37.2 \mathrm{~mm}^{2}$.

- Mean magnetic path length $l_{c}=28.72 \mathrm{~mm}$.
- Relative permeability of the core material $\mu_{r c}=3000$.

In addition, in order to avoid distortion due to core saturation, the maximum choke inductor current is assumed as For $I_{L f \max }=1.2 \mathrm{~A}$. Therefore, using (22), the total area of the winding is

$$
A_{w}=A_{w(s o l)}=\frac{I_{L f(\max )}}{J_{m}}=\frac{1.2}{5 \times 10^{6}}=0.24 \mathrm{~mm}^{2} .
$$

From the AWG datasheet, the selected winding is AWG 23. The inner diameter of the selected wire is $d_{i}(s o l)=$ $0.573 \mathrm{~mm}$, the outer diameter is $d_{o(s o l)}=0.632 \mathrm{~mm}$, and the bare wire area is $A_{w(s o l)}=0.258 \mathrm{~mm}^{2}$.

Using (13), the window area is $W_{a}=13.45 \mathrm{~mm}^{2}$. Therefore, $W_{a p}=W_{a} / 2=6.72 \mathrm{~mm}^{2}$. From (14), the number of turns is

$$
N=\frac{K_{u} W_{a}}{A_{w(\text { sol })}}=\frac{0.4 \times 6.725 \times 10^{-6}}{0.258 \times 10^{-6}}=10.38 .
$$

In comparison, using (15), the number of turns assuming $l_{g}=$ $0.1 \mathrm{~mm}$ is

$$
\begin{aligned}
N & =\sqrt{\frac{L_{f}}{\mu_{0} A_{c}}\left(l_{g}+\frac{l_{c}}{\mu_{r c}}\right)} \\
& =\sqrt{\frac{40 \times 10^{-6} \times\left(0.1+9.567 \times 10^{-3}\right) \times 10^{-3}}{4 \pi \times 10^{-7} \times 37.2 \times 10^{-6}}}=9.68
\end{aligned}
$$

Thus, using (16), the number of turns chosen for this design is

$$
N=\max \{10.38,9.68\}=10.38 \text {. }
$$

Select the number of turns as $N=10$. The maximum thickness of the winding is equal to the diameter of the solid round wire, $d_{i(s o l)}=0.552 \mathrm{~mm}$. From the core datasheet, the height of the window for the selected core geometry is $H=2 D=7.4 \mathrm{~mm}$. For 10 turns, the overall height occupied by the windings is $H=N d_{i(s o l)}=5.52<2 D$. Therefore, the winding arrangement consists of only one layer, i.e., $N_{l}=1$. Further, the wire length per single turn wound around the center-post of the selected core is

$$
l_{T}=\pi F=\pi \times 7.45 \times 10^{-3}=23.40 \mathrm{~mm} .
$$

Therefore, the total length of the wire is $l_{w}=N l_{T}=0.234 \mathrm{~m}$. For the given core and electrical parameters, the peak value of the magnetic flux density for the gapped core as given in (17) is

$$
\begin{aligned}
B_{p k} & =\frac{\mu_{0} \mu_{r c} N I_{L f(\max )}}{l_{c}+\mu_{r c} l_{g}} \\
& =\frac{4 \pi \times 10^{-7} \times 3000 \times 10 \times 1.2}{\left(28.72 \times 10^{-3}\right)+\left(3000 \times 0.1 \times 10^{-3}\right)} \\
& =0.137 \mathrm{~T} .
\end{aligned}
$$

For the selected core material, the saturation flux density is $B_{\text {sat }}=0.45 \mathrm{~T}$ at $25^{\circ} \mathrm{C}$ and $B_{p k}<B_{\text {sat }}$. Thus, the chosen air gap avoids core saturation. The solid round winding has a single layer wound over the center post of the selected core. 
From the fundamental harmonic approximation discussed in Section II-A, the triangular waveform of the ac component of the choke inductor current is approximated by its fundamental sinusoidal component. The amplitude of the fundamental of ac component through the choke inductor is

$$
I_{L f m 1}=\frac{8}{\pi^{2}} I_{L f m}=0.8105 \times 0.0625=0.0506 \mathrm{~A}
$$

The amplitude of the sinusoidal magnetic flux density using (18) is

$$
\begin{aligned}
B_{c m} & =\frac{\mu_{0} \mu_{r c} N I_{L f m 1}}{l_{c}+\mu_{r c} l_{g}} \\
& =\frac{4 \pi \times 10^{-7} \times 3000 \times 10 \times 0.0506}{\left(28.72 \times 10^{-3}\right)+\left(3000 \times 0.1 \times 10^{-3}\right)} \\
& =5.77 \mathrm{mT} .
\end{aligned}
$$

\section{B. Power Losses in the Choke}

For the selected core material and the specified operating frequency $f=f_{s}=1000 \mathrm{kHz}$, the Steinmetz parameters are $k=7.36 \times 10^{-7}, a=3.47$, and $b=2.54$. Thus, the total core power loss density for $B_{c m}=5.77 \mathrm{mT}$ using (19) is

$$
\begin{aligned}
P_{v g} & =k f^{a}\left(B_{c m}\right)^{b} \\
& =7.36 \times 10^{-7} \times\left(1 \times 10^{3}\right)^{3.47} \times\left(5.77 \times 10^{-3}\right)^{2.54} \\
& =0.0389 \frac{\mathrm{mW}}{\mathrm{cm}^{3}} .
\end{aligned}
$$

The volume of the core $V_{C}=A_{c} l_{c}=37.2 \times 28.72=$ $1.068 \mathrm{~cm}^{3}$. Therefore, the total core power loss using (20) is

$$
P_{C}=P_{v g} V_{C}=0.0389 \frac{\mathrm{mW}}{\mathrm{cm}^{3}} \times 1.068 \mathrm{~cm}^{3}=0.0415 \mathrm{~mW} \text {. }
$$

For the copper winding, the skin depth is estimated using (21) as

$$
\delta_{w}=\frac{66.2}{\sqrt{f_{s}}}=\frac{66.2}{\sqrt{10^{6}}}=0.0662 \mathrm{~mm} .
$$

The dc resistance of the solid round winding is

$$
R_{w d c(s o l)}=\frac{4 \rho N l_{T}}{\pi d_{i(s o l)}^{2}}=16.8 \mathrm{~m} \Omega
$$

yielding the conduction winding loss due to the dc current as

$$
P_{R w d c(s o l)}=I_{L f}^{2} R_{w d c(s o l)}=20.328 \mathrm{~mW} .
$$

The ac resistance of the solid round winding is

$$
R_{w a c(s o l)}=\frac{4 \rho N l_{T}}{\delta_{w}\left(d_{i}-\delta_{w}\right)}=0.048 \Omega .
$$

The power loss due to the ac component

$$
P_{w a c(s o l)}=\frac{I_{m 1}^{2}}{2} R_{w a c(s o l)}=0.0615 \mathrm{~mW} \text {. }
$$

The ratio of the winding de power loss to the winding ac power loss is

$$
\frac{P_{R w d c(s o l)}}{P_{w a c(s o l)}}=331
$$

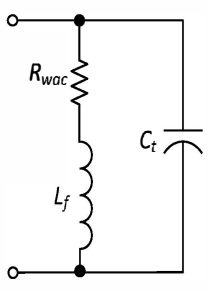

Fig. 4. High-frequency lumped-circuit model of a choke inductor

One can observe that the winding dc power loss is dominated by the power loss due to dc component of the choke inductor current. Since the number of layers $N_{l}=1$, the proximity effect is absent. Thus, neglecting the ac winding loss, the total power loss in the choke inductor is

$P_{L f(s o l)}=P_{C g}+P_{R w d c(s o l)}=0.04+20.328=20.368 \mathrm{~mW}$

\section{AC Characteristics of Choke Inductor}

The high-frequency model of choke inductor is shown in Fig. 4. The turn-to-turn capacitance $C_{t t}$ formed by the conductor turns of a single-layer inductor is expressed as [6]

$$
C_{t t}=\frac{2 \epsilon_{0} l_{T}}{\sqrt{\left(1+\frac{1}{\epsilon_{r}} \ln \frac{D_{w 0}}{D_{w i n}}\right)^{2}-1}} \arctan \left(\sqrt{\frac{2+\frac{1}{\epsilon_{r}} \ln \frac{D_{w \bullet}}{D_{w i n}}}{\frac{1}{\epsilon_{r}} \ln \frac{D_{w 0}}{D_{w i n}}}}\right) .
$$

The equivalent capacitance $C_{t}$ for $N$ turns assuming the turnto-turn capacitances are in series is given as $C_{t}=\frac{C_{t t}}{N-1}$. At high frequencies, the displacement current bypasses the inductance and flows through the capacitance. The resistance $R_{w a c}$ is the ac series resistance. The impedance of the model shown in Fig. 4 is

$$
Z=\left(R_{w a c}+s L_{f}\right) \| \frac{1}{s C_{t}}=R_{w a c} \frac{1+\frac{s}{\omega_{z i}}}{1+\frac{s}{Q_{\bullet} \omega_{\bullet}}+\left(\frac{s}{\omega_{\bullet}}\right)^{2}},
$$

where the frequency of the zero is $\omega_{z i}=\frac{R_{\text {wac }}}{L_{f}}$, the selfresonant frequency is $f_{0}=\frac{1}{2 \pi \sqrt{L_{f} C_{t}}}$, and the quality factor of the choke is $Q_{0}=\frac{1}{2 \xi}=\frac{1}{R_{w a c}} \sqrt{\frac{L_{f}}{C_{t}}}$. Fig. 5 shows the magnitude of the impedance of the high-frequency model of choke inductor as a function of frequency. Fig. 6 shows the phase of the impedance of the high-frequency model of choke inductor as a function of frequency.

\section{Simulation RESUlts}

The components in the Class-E amplifier component were designed using the procedure presented in [1]. The values of the circuit components are: choke inductance $L_{f}=40 \mu \mathrm{H}$ (as calculated earlier), shunt capacitor $C_{1}=5.1 \mathrm{nF}$, resonant capacitor $C=4.7 \mathrm{nF}$, resonant inductor $L=6.5 \mu \mathrm{H}$, and load resistor $R=6 \Omega$. The circuit was built on SABER circuit simulator. Fig. 7 shows the results obtained by transient analysis. For the designed circuit, the maximum current $I_{L f(\max )}$ 


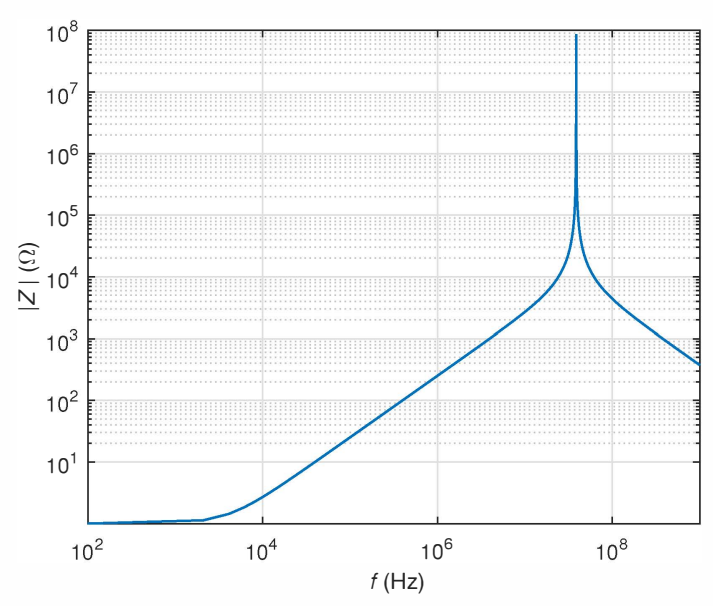

Fig. 5. Impedance magnitude as a function of frequency.

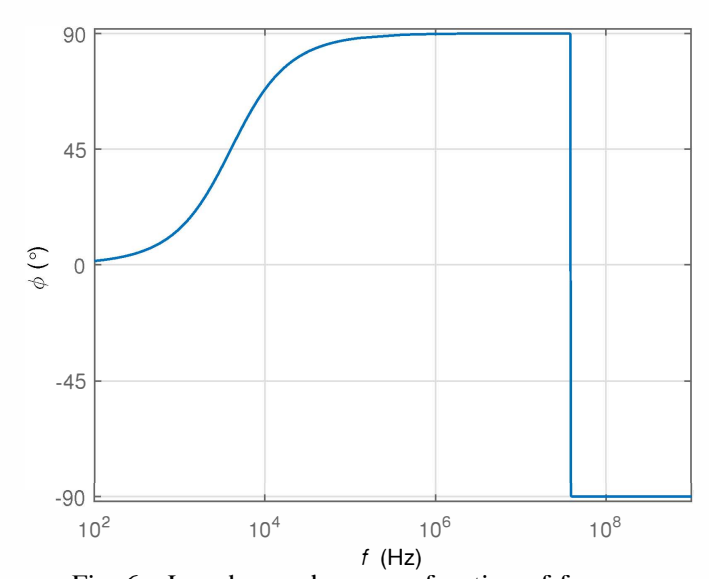

Fig. 6. Impedance phase as a function of frequency.

was recorded as $1.171 \mathrm{~A}$. The average value was measured as $I_{I}=I_{L f}=1.0925 \mathrm{~A}$. The ac magnitude was measured as $I_{L f m}=0.0618 \mathrm{~A}$. The power loss in the choke inductor was measured and is equal to $P_{L f}=0.15 \mathrm{~W}$. From the Fourier analysis result shown in Fig. 8, it was noted that the the dc component of the choke inductor current was dominant in comparison with the fundamental component and consecutive harmonic components. Therefore, one may conclude that the power loss in the choke is primarily due to the dc current.

\section{CONCLUSIONS}

This paper has presented the design procedure for the choke used in Class-E ZVS power amplifiers using area product method. A gapped core has been selected to avoid core saturation. The power loss has been evaluated through a modified Steinmetz equation for gapped cores. The core losses are low because the core is air gapped and the ac component of the current is also low and results in a limited ac component of the flux density. The ac resistance of solid round winding have been determined using the effective area available for current conduction at high operating frequencies. The dc and ac winding power losses due to the dc and ac fundamental

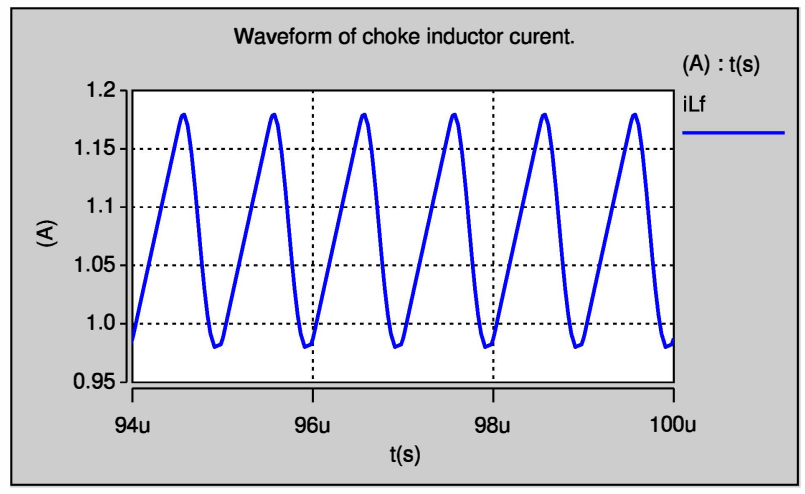

Fig. 7. Waveform of the choke inductor current $i_{L f}$ obtained through SABER.

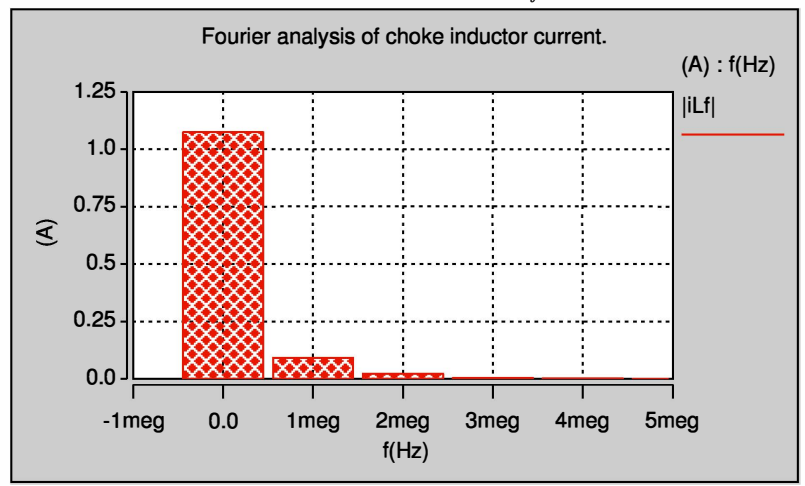

Fig. 8. Frequency spectrum of the choke inductor current obtained through SABER showing its dominant dc component.

choke current components have been determined. It has been shown that the ac winding loss is significantly lower than the dc winding loss and can be neglected for the design presented in this paper. The dc winding loss is also higher than the core power loss for this design example. Thus, the power loss due to the dc choke inductor current is the dominant loss component. Simulation results have been presented to show the frequency spectrum of the choke inductor current. The Fourier analysis result has revealed that the dc component of the current through the choke is dominant compared to the fundamental and the consecutive harmonics. Therefore, the power loss due to the harmonics in the choke inductor current can be neglected. The self-capacitance has been determined and can be included by the shunt capacitor of the amplifier.

\section{REFERENCES}

[1] M. K. Kazimierczuk, Radio-Frequency Power Amplifiers, 2nd. Ed., Wiley, Chichester, UK, 2015.

[2] M. K. Kazimierczuk and H. Sekiya, "Design of ac resonant inductors using area product method," in IEEE Energy Conversion Congress and Exhibition, San Jose, CA, Sept. 2009, pp. 994-1001.

[3] M. K. Kazimierczuk, High-Frequency Magnetic Components, 2nd. Ed., Wiley, Chichester, UK, 2014.

[4] C. P. Steinmetz, "On the law of hysteresis,", Proc. IEEE, vol. 72, pp. $197221,1984$.

[5] A. Ayachit and M. K. Kazimierczuk, "Steinmetz equation for gapped magnetic cores," IEEE Magnetics Letters, vol. 7, May 2016.

[6] S. W. Pasko, M. K. Kazimierczuk, and B. Grzesik, "Self-capacitance of coupled toroidal inductor for EMI filters," IEEE Trans. Electromagnetic Compat., vol. 57, no. 2, pp. 216-223, Apr. 2015.

[7] American Wire Gauge (AWG) Datasheet. https://www.micrometals.com.

[8] Magnetics Ferrite Cores, 2013 Catalog. htttps://www.mag-inc.com. 